\title{
Paecilomyces cateniannulatus Liang, a commonly found, but an unrecorded entomogenous fungus in Japan
}

\author{
Mitsuaki Shimazu \\ Forestry and Forest Products Research Institute, Tsukuba, Ibaraki 305-8687, Japan
}

(Received 31 January 2001; Accepted 20 April 2001)

\begin{abstract}
Cultural and morphological characters of isolates belonging to an entomogenous Paecilomyces species commonly found in Japan were investigated and compared with the literature. This species has not been recorded in major monographs of this genus, but identified as Paecilomyces cateniannulatus Liang described in 1981 from China. Mycelia on insects and media were pure white, and sometimes produce loose synnemata up to $10 \mathrm{~mm}$. Growth rate of the fungus is moderate. The reverse side of colonies on SDY or MEA media are colorless to light yellow. Phialides are flask shaped with a narrow neck, 3.0-16.1 $\times 1.3-3.2 \mu \mathrm{m}$, often forming a whorl. Conidia are oval to short cylindrical, $2.1-$ $4.6 \times 1.3-2.4 \mu \mathrm{m}$, arranged in basipital chains. Conidial chains are often irregularly curved and sometimes form a loop. Early stage of the conidiogenous structure of this fungus resembles that of Beauveria, but is distinguished by formation of conidial chains.
\end{abstract}

Key words: Paecilomyces cateniannulatus, entomogenous fungi, new record

\section{INTRODUCTION}

The hyphomycete genus Paecilomyces includes more than 13 entomogenous species (Samson et al., 1988; Aoki, 1989). Among collections of entomopathogenic fungi in the Insect Pathology Laboratory of the Forestry and Forest Products Research Institute, about 40 isolates have been identified as species belonging to the genus Paecilomyces. This particular fungus is not unusual in Japan, but sometimes important as a natural enemy of forest pest insects (Ando, 1985) or a microbial control agent (Mitsuhashi et al., 1992). However, the taxonomic state of this fungus has not been clarified, and moreover, this species has not been mentioned in major monographs of the genus Paecilomyces or related genera written by Brown and Smith (1957) and Samson (1974). Consulting the literature, Paecilomyces cateniannulatus Liang (Liang, 1981) seems to have characteristics identical with our fungus. Here, the characters of this fungus are described for the convenience of identification.

\section{MATERIALS AND METHODS}

Thirty isolates of Paecilomyces from insects, soil and plant leaves in the entomogenous fungus collection at the Forestry and Forest Products Research Institute thought to belong to the same species were examined (Table 1). The isolates from soils and leaves were obtained by using $\mathrm{D} 0 \mathrm{C} 2$ medium (Bactopeptone $3 \mathrm{~g}, \mathrm{CuCl}_{2} 0.2 \mathrm{~g}$, crystal violet $2 \mathrm{mg}$ and agar $15 \mathrm{~g}$, in $1,000 \mathrm{ml}$ of distilled water, $\mathrm{pH}$ 10.0; Shimazu and Sato, 1996). This medium was developed for selective isolation of Beauveria bassiana, but some other entomogenous Deuteromycetes can also grow on it.

For the measurements of conidia and phialides, these isolates were innoculated on Sabouraud's dextrose agar medium with $1 \%$ yeast extract (SDY) in plastic petri dishes $55 \mathrm{~mm}$ in diameter, and cultured at $25^{\circ} \mathrm{C}$ for 14 to 17 days under continuous lighting with fluorescent lamps. Each isolate was also cultured on a sterilized glass slide with a piece of SDY to investigate conidiogenous structures.

Conidia and phialides taken from the cultures were mounted in an aqueous solution of $0.85 \%$ $\mathrm{NaCl}$ with $3.5 \%$ formaldehyde (PSF) on glass slides. To check the influence of mounting fluids on conidial dimensions, conidial specimens taken from 2 isolates were also mounted in lactophenolcotton blue (LCB). Lengths and widths of conidia were measured on a TV screen connected to a microscope, and converted to the actual sizes.

SDY medium and malt extract agar medium (malt extract $20 \mathrm{~g}$, Bactopeptone $1 \mathrm{~g}$, dextrose $20 \mathrm{~g}$, and agar $15 \mathrm{~g}$ in $1,000 \mathrm{ml}$ of distilled water, abbreviation MEA) were used to investigate hyphal 
Table 1. Isolates of Paecilomyces cateniannulatus examined in this experiment

\begin{tabular}{|c|c|c|}
\hline Isolate number & Origin & Location \\
\hline $\mathrm{F}-430$ & Resseliella odai (Diptera; Cecidomiidae) larva & Nakatsue, Oita \\
\hline F-594 & Epinotia granitalis (Lepidoptera; Tortricidae) larva & Yasato, Ibaraki \\
\hline F-630 & Galleria mellonella (Lepidoptera; Pyralidae) larva buried in soil & Kukisaki, Ibaraki \\
\hline F-648 & Epinotia granitalis (Lepidoptera; Tortricidae) larva & Yasato, Ibaraki \\
\hline F-658 & Morophagoides moriutii (Lepidoptera; Tineidae) pupa & Kukisaki, Ibaraki \\
\hline F-724 & Morophagoides moriutii (Lepidoptera; Tineidae) larva & Kukisaki, Ibaraki \\
\hline F-803 & Morophagoides moriutii (Lepidoptera; Tineidae) pupa & Lab-reared \\
\hline F-804 & Morophagoides moriutii (Lepidoptera; Tineidae) pupa & Lab-reared \\
\hline F-870 & Morophagoides moriutii (Lepidoptera; Tineidae) larva & Sanbu, Chiba \\
\hline F-871 & Morophagoides moriutii (Lepidoptera; Tineidae) larva & Ichinomiya, Chiba \\
\hline F-872 & Epinotia granitalis (Lepidoptera; Tortricidae) larva & Kiryu, Gunma \\
\hline F-891 & Lepidopteran pupa in soil & Kukisaki, Ibaraki \\
\hline F-923 & Homona magnanima (Lepidoptera; Tortricidae) larva & Hamamatsu, Shizuoka \\
\hline F-988 & Lepidopteran pre-pupa in soil & Ootsu, Shiga \\
\hline F-1132 & Agriotypus sp. (Hymenoptera; Ichneumonidae) & Kawachinagano, Osaka \\
\hline F-1186 & unknown insect under Quercus bark & Yasato, Ibaraki \\
\hline F-1188 & unknown insect in a cocoon made of dead Cryptomeria needles & Yasato, Ibaraki \\
\hline F-1189 & unknown insect under Quercus bark & Yasato, Ibaraki \\
\hline F-1190 & unknown insect in moss & Yasato, Ibaraki \\
\hline F-1201 & unknown insect in moss & Kumamoto, Kumamoto \\
\hline F-1271 & Ceruchus lignarus (Coleoptera; Lucanidae) larva & Lab-reared \\
\hline F-1283 & Lepidopteran pupa in soil & Tsukuba, Ibaraki \\
\hline F-1308 & Monochamus alternatus (Coleoptera; Cerambycidae) adult & Lab-reared \\
\hline F-1385 & on a leaf of Houttuynia cardata (Piperales; Saururaceae) & Kumamoto, Kumamoto \\
\hline F-1389 & on a leaf of Morus bombycis (Urticales; Moraceae) & Kumamoto, Kumamoto \\
\hline F-1390 & on a leaf of Morus bombycis (Urticales; Moraceae) & Kumamoto, Kumamoto \\
\hline F-1461 & directly isolated from soil & Kukisaki, Ibaraki \\
\hline F-1462 & directly isolated from soil & Kukisaki, Ibaraki \\
\hline F-1507 & Lepidopteran pupa in soil & Hachioji, Tokyo \\
\hline F-1509 & Lepidopteran pupa in soil & Ushiku, Ibaraki \\
\hline
\end{tabular}

growth of this fungi at various temperatures. The pHs of both media were adjusted to 6.5 with $\mathrm{HCl}$ for SDY and $\mathrm{Na}_{2} \mathrm{CO}_{3}$ for MEA. The media were prepared in $9 \mathrm{~cm}$ petri dishes. As inoculum for the agar plates, the fungus was cultured in Sabouraud's saccharose broth with $1 \%$ yeast extract in a shaker incubator to produce a suspension of hyphal bodies. A drop of the suspension was inoculated at the center of each agar plate with a platinum loop and dried. The plates were then incubated at 10, 15, 20, 25 , and $30^{\circ} \mathrm{C}$ in total darkness. For each temperature, 4 plates were replicated. The plates were incubated for 14 days and the 2 perpendicularly intersecting diameters of each colony were measured.

\section{RESULTS}

On insect bodies, mycelia of the fungus were pure white and cottony, and often produced soft simple synnemata (Fig. 1A). Colonies of all isolates on SDY agar were also pure white and cottony. Some produced many loose and soft synnemata up to $10 \mathrm{~mm}$ (Fig. 1B). Usually, conidia were produced sparsely, and consequently, appearances of colonies were not powdery even after maturation. Reverse sides of the dishes were generally colorless to bright yellow.

General microscopic morphology is illustrated in Fig. 2, which was traced from the microscope TV screen. Phialides were flask shaped with a narrow neck, 4.1-11.7 $\times 1.5-2.7 \mu \mathrm{m}$, often arranged in a whorl (Fig. 3A), but, sometimes borne solitary (Fig. 3B). Conidia were hyaline, smooth walled, oval to short cylindrical, both ends round, 2.1$4.6 \times 1.3-2.4 \mu \mathrm{m}$, the length/width ratios were 1.2 2.7 when mounted in PSF (Table 2, Fig. 3C). When mounted in LCB, they were $1.8-2.7 \times 1.3-1.6 \mu \mathrm{m}$, $\mathrm{L} / \mathrm{W}$ ratio $1.2-2.0$. Conidial sizes mounted in LCB 

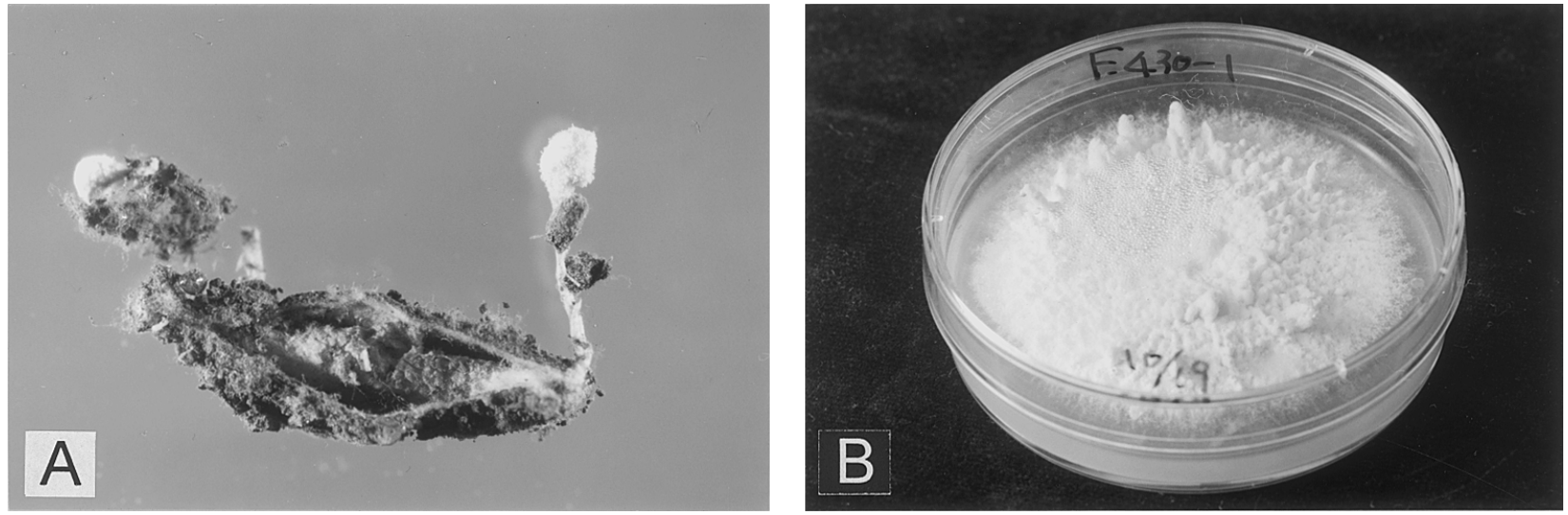

Fig. 1. Appearances of Paecilomyces cateniannulatus on a lepidopteran pupa (A) and on SDY agar medium (B).
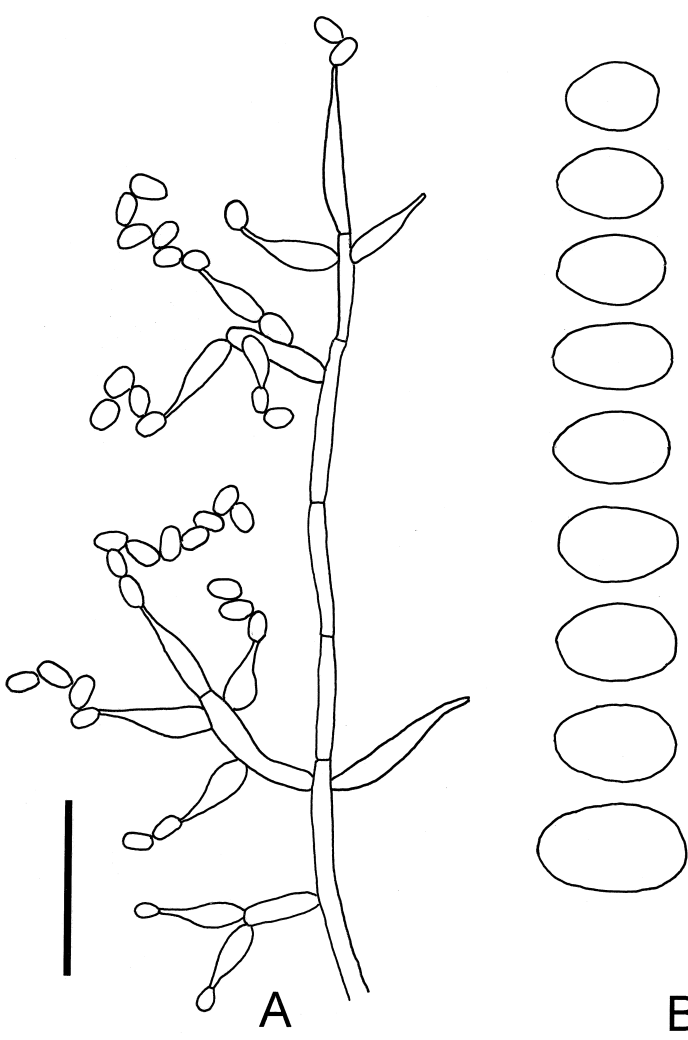

B

Fig. 2. Conidiogenous structures of Paecilomyces cateniannulatus; A: phialides and conidial chains, B: conidia (scale bars: $10 \mu \mathrm{m})$.

were approximately $80 \%$ of those mounted in PSF. Conidia were arranged in basipital chains. In the chains, conidia contacted each other not always by their ends but often by sides, consequently the conidial chains often become irregularly curved or sometimes formed a loop. When shaker-cultured in liquid media, this fungus formed hyphal bodies similar to that seen in insect hemolymph (Fig. 3D).
The fungus grew at all temperatures examined. The best growth was observed at $25^{\circ} \mathrm{C}$ for both media, while growth was the poorest at $30^{\circ} \mathrm{C}$ (Fig. 4). The differences among the colony diameters at various temperatures were greater on MEA than on SDY. The average diameter on MEA at $25^{\circ} \mathrm{C}$ day 14 was $51 \mathrm{~mm}$ while that on SDY was $41 \mathrm{~mm}$. The growth rate of this fungus on SDY was approximately the same as that of other entomogenous Deuteromycetes (Sato et al., 1993).

\section{DISCUSSIONS}

Although isolates used in the present study were obtained from various taxa of insects, leaves and even from soils, they have the same morphological features, and are thought to belong to a single species. The conidiogenous structure with whorls of phialides and conidial chains indicates that this species belongs to the genus Paecilomyces. One unique feature of the present fungus is that it forms irregularly curved conidial chains. However, the chains are not orderly oblique like that of Mariannaea (Samson, 1974; Liang, 1991). Among the Paecilomyces species, Paecilomyces farinosus and Paecilomyces cateniannulatus have similar conidial sizes and colors to the present fungus (Samson, 1974; Liang, 1981). Conidia of $P$. farinosus are fusiform to lemon-shaped with pointed ends, and arranged in a regular conidial chain typical of this genus. On the other hand, those of $P$. cateniannulatus are oval to ellipsoidal, lie broadside on one another and slime down to form a ring or irregular masses. Features of the present fungus coincide with those of $P$. cateniannulatus, and this species 

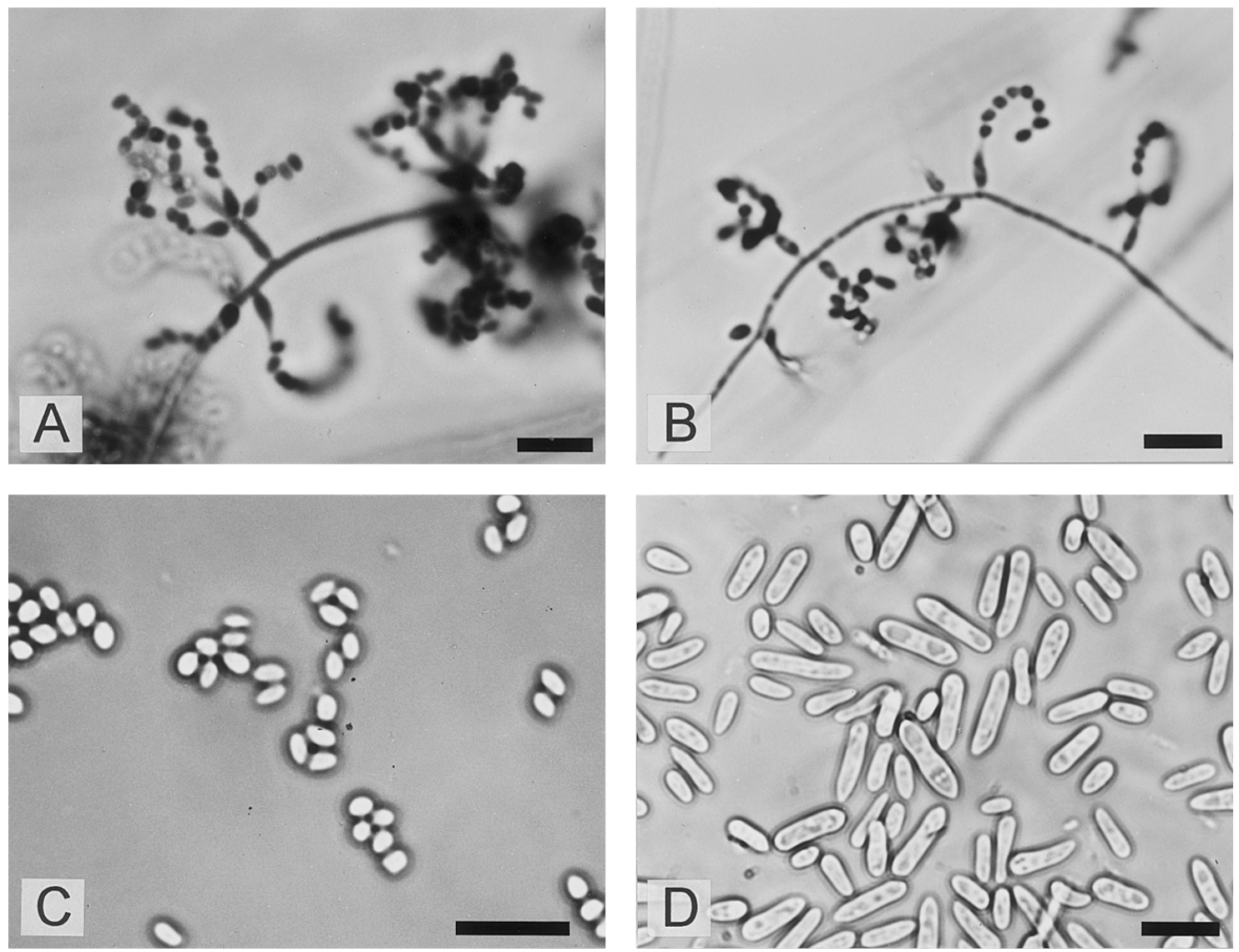

Fig. 3. Microscopical morphology of Paecilomyces cateniannulatus; A: phialides arranged in a whorl stained with LCB, B: solitary phialides stained with LCB, C: conidia in PSF solution, D: hyphal bodies in SDY broth (scale bars: $10 \mu \mathrm{m}$ ).

was identified as $P$. cateniannulatus. Another species, Paecilomyces cateniobliquus, also produces irregular conidial chains. However, colonies of this species are pink to deep rose color with red synnemata, and its conidia are larger than P. cateniannulatus (Liang, 1991).

According to the original description, this fungus was isolated from an adult coleopteran species and the pupa of Cacoecia ingentana (=Archips ingentana), Lepidoptera. In the present study, isolates of this species were from Coleoptera, Diptera, Hymenoptera, Lepidoptera, and moreover, from non-insect origins such as tree bark, leaves, or soil. Since this fungus has only been reported from China and Japan, and not from Europe, America or other areas, this fungus may be unique to East Asia.

Phialides of this species sometimes resemble the conidiogenous cells of Beauveria. Arx (1986) combined Tolypocladium within Beauveria, based on morphological characters of the conidiogenous cells, however, this viewpoint has not been widely accepted because of differences in the conidiogenous orders. The flask shaped base and slender neck of a Tolypocladium phialide certainly resemble a sympodula of Beauveria, and their appearances on media also appear similar. Samson and Soares (1984) pointed out that Tolypocladium species might have been misidentified as Beauveria species due to their similarities. Similarly, the present fungus, $P$. cateniannulatus also has a phialide with a slender neck and an inflated base, and the cultures appear pure white, thus, this species may also have been confused with Beauveria. Especially, at its early stage of sporulation when only 1 or 2 conidia are formed, this species could be misidentified as Beauveria (Fig. 5A, B). Thus the present fungus may also be confused with Tolypocladium species. However, the present fungus can be distinguished by dry conidial chain and faster growth on MEA while Tolypocladium species produce conidia in mucus and grow very 
Table 2. Conidial dimensions of Paecilomyces cateniannulatus isolates

\begin{tabular}{|c|c|c|c|c|c|c|c|c|c|}
\hline \multirow{2}{*}{ Isolate No. } & \multicolumn{3}{|c|}{ Length $(\mu \mathrm{m})$} & \multicolumn{3}{|c|}{ Width $(\mu \mathrm{m})$} & \multicolumn{3}{|c|}{ Length/width } \\
\hline & Max. & Mean & Min. & Max. & Mean & Min. & Max. & Mean & Min. \\
\hline F-430 & 3.1 & 2.8 & 2.4 & 1.9 & 1.7 & 1.4 & 1.9 & 1.6 & 1.4 \\
\hline F-594 & 3.1 & 2.8 & 2.4 & 2.3 & 1.9 & 1.6 & 1.8 & 1.5 & 1.2 \\
\hline F-630 & 3.3 & 2.8 & 2.4 & 1.9 & 1.6 & 1.3 & 2.1 & 1.7 & 1.4 \\
\hline F-648 & 3.1 & 2.7 & 2.3 & 2.4 & 1.8 & 1.4 & 1.8 & 1.5 & 1.2 \\
\hline F-658 & 3.3 & 2.8 & 2.6 & 1.8 & 1.6 & 1.4 & 1.9 & 1.7 & 1.5 \\
\hline F-724 & 3.1 & 2.7 & 2.2 & 1.8 & 1.5 & 1.3 & 1.9 & 1.7 & 1.5 \\
\hline F-803 & 3.7 & 3.0 & 2.5 & 1.8 & 1.7 & 1.4 & 2.1 & 1.8 & 1.6 \\
\hline F-804 & 3.1 & 2.8 & 2.4 & 2.0 & 1.7 & 1.4 & 1.9 & 1.6 & 1.2 \\
\hline F-870 & 3.4 & 2.9 & 2.5 & 1.8 & 1.7 & 1.4 & 2.1 & 1.7 & 1.5 \\
\hline F-871 & 3.4 & 3.0 & 2.7 & 2.1 & 1.8 & 1.7 & 2.0 & 1.7 & 1.4 \\
\hline F-872 & 4.6 & 3.0 & 2.4 & 2.3 & 1.8 & 1.7 & 2.5 & 1.7 & 1.3 \\
\hline F-891 & 3.8 & 2.9 & 2.4 & 2.1 & 1.7 & 1.3 & 2.1 & 1.8 & 1.5 \\
\hline F-923 & 3.9 & 3.1 & 2.5 & 2.0 & 1.8 & 1.6 & 2.4 & 1.8 & 1.3 \\
\hline F-988 & 3.8 & 3.1 & 2.7 & 2.3 & 2.1 & 1.9 & 1.8 & 1.5 & 1.3 \\
\hline F-1132 & 3.6 & 2.9 & 2.5 & 2.0 & 1.8 & 1.5 & 2.4 & 1.7 & 1.4 \\
\hline F-1186 & 3.5 & 2.9 & 2.4 & 2.1 & 1.7 & 1.4 & 2.2 & 1.8 & 1.3 \\
\hline F-1188 & 4.0 & 2.9 & 2.3 & 2.0 & 1.8 & 1.5 & 2.0 & 1.6 & 1.4 \\
\hline F-1189 & 3.8 & 2.9 & 2.4 & 2.0 & 1.7 & 1.4 & 2.0 & 1.7 & 1.4 \\
\hline F-1190 & 3.8 & 3.2 & 2.5 & 2.0 & 1.7 & 1.3 & 2.7 & 1.9 & 1.5 \\
\hline F-1201 & 2.8 & 2.4 & 2.1 & 1.9 & 1.6 & 1.3 & 1.6 & 1.5 & 1.3 \\
\hline F-1271 & 3.0 & 2.7 & 2.2 & 2.1 & 1.8 & 1.6 & 1.7 & 1.5 & 1.2 \\
\hline F-1283 & 3.4 & 2.9 & 2.4 & 2.0 & 1.8 & 1.6 & 1.9 & 1.6 & 1.4 \\
\hline F-1308 & 3.8 & 2.8 & 2.2 & 2.1 & 1.7 & 1.5 & 2.0 & 1.7 & 1.3 \\
\hline F-1385 & 3.0 & 2.6 & 2.2 & 2.0 & 1.7 & 1.5 & 1.8 & 1.5 & 1.4 \\
\hline F-1389 & 3.3 & 2.8 & 2.4 & 2.0 & 1.7 & 1.5 & 1.9 & 1.6 & 1.4 \\
\hline F-1390 & 3.1 & 2.7 & 2.3 & 1.9 & 1.7 & 1.5 & 1.9 & 1.6 & 1.4 \\
\hline F-1461 & 3.9 & 3.1 & 2.7 & 2.2 & 1.8 & 1.7 & 2.2 & 1.7 & 1.4 \\
\hline F-1462 & 3.5 & 2.9 & 2.5 & 1.9 & 1.7 & 1.3 & 2.0 & 1.7 & 1.5 \\
\hline F-1507 & 3.4 & 3.2 & 2.7 & 2.2 & 1.9 & 1.6 & 1.9 & 1.7 & 1.4 \\
\hline F-1509 & 3.5 & 3.1 & 2.7 & 2.2 & 2.0 & 1.6 & 2.1 & 1.6 & 1.3 \\
\hline Max. & 4.6 & 3.2 & 2.7 & 2.4 & 2.1 & 1.9 & 2.7 & 1.9 & 1.6 \\
\hline Mean & 3.5 & 2.9 & 2.4 & 2.0 & 1.7 & 1.5 & 2.0 & 1.7 & 1.4 \\
\hline Min. & 2.8 & 2.4 & 2.1 & 1.8 & 1.5 & 1.3 & 1.6 & 1.5 & 1.2 \\
\hline
\end{tabular}

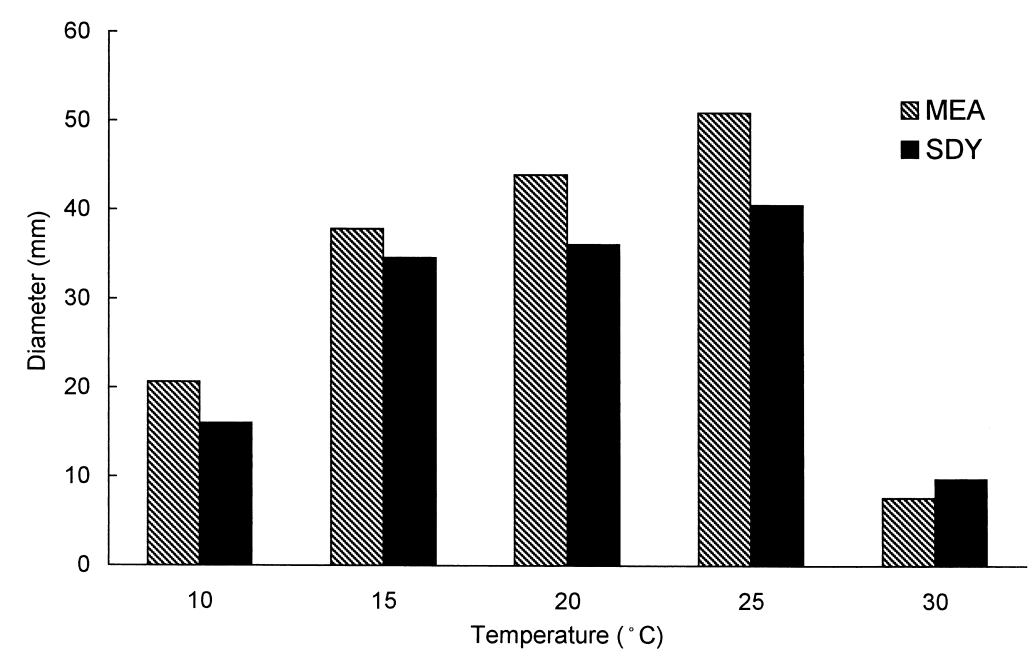

Fig. 4. Mycelial growth of Paecilomyces cateniannulatus F-430 on SDY and MEA media on day 14. 

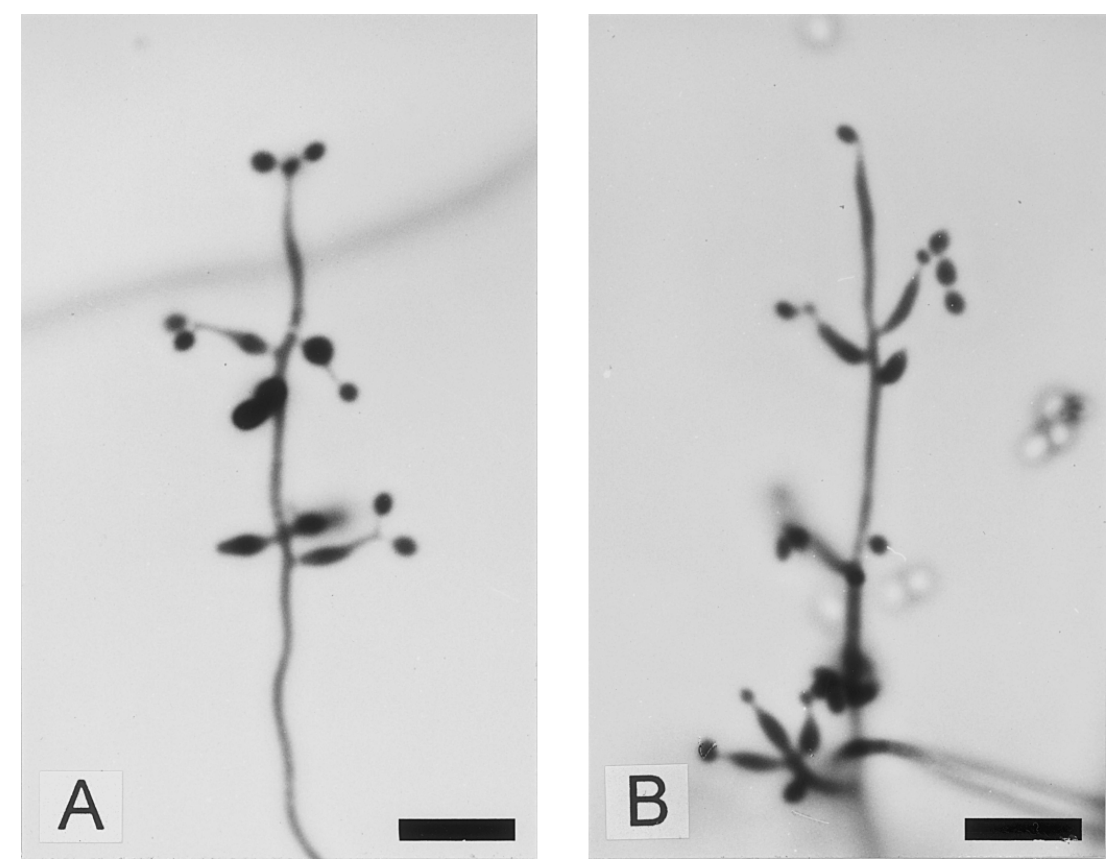

Fig. 5. Similarities of conidiogenous structures in early stages of Beauveria bassiana (A) and Paecilomyces cateniannulatus (B) (scale bars: $10 \mu \mathrm{m})$.

slowly on MEA (Samson and Soares, 1984).

This fungus has been isolated from various species of insects, and is thought to be pathogenic to various insects. According to Ando (1985), this fungus was found to be an important natural enemy causing more than $90 \%$ mortality in the cryptomeria pitch midge, Resseliella odai. Mitsuhashi et al. (1992) mass-produced this fungus and Paecilomyces fumosoroseus, and applied them to the bark of Cryptomeria for control of Epinotia granitalis, and obtained a mortality of $61 \%$. The potential of this fungus as a microbial control agent for other insect pests is promising.

\section{REFERENCES}

Ando, S. (1985) [Paecilomyces sp. attacking cryptomeria pitch midge, Resseliella odai.] Trans. 38th Meet. Kyushu Branch Jpn. For. Soc., 181-182 (in Japanese).

Aoki, J. (1989) [A Key to Insect Pathogenic Fungi.] Zenkoku Noson Kyoiku Kyokai, Tokyo. 280 pp. (in Japanese).

Arx, J. A. von (1986) Tolypocladium, a synonym of Beauveria. Mycotaxon 25: 153-158.

Brown, A. H. S. and G. Smith (1957) The genus Paecilomyces Bainier and its perfect stage Byssochlamys Westling.
Trans. Br. Mycol. Soc. 40: 17-89.

Liang, Z.-q. (1981) Two new species of Paecilomyces from insects. Acta Microbiol. Sin. 21: 31-34 (in Chinese with English summary).

Liang, Z.-q. (1991) Verification and identification of the anamorph of Cordyceps pruinosa Petch. Acta Mycol. Sin. 10: 104-107 (in Chinese with English summary).

Mitsuhashi, W., M. Shimazu and H. Hashimoto (1992) Control of Epinotia granitalis (Lepidoptera: Tortricidae) with Paecilomyces spp. on cotton bands wrapped on the trunks of Cryptomeria japonica. Appl. Entomol. Zool. 27: 295296.

Samson, R. A. (1974) Paecilomyces and some allied Hyphomycetes. Stud. Mycol. 6: 1-119.

Samson, R. A., H. C. Evans and J. P. Latgé (1988) Atlas of Entomopathogenic Fungi. Springer-Verlag, Berlin. 187 pp.

Samson, R. A. and G. G. Soares, Jr. (1984) Entomopathogenic species of the Hyphomycete genus Tolypocladium. J. Invertebr. Pathol. 43: 133-139.

Sato, H., W. Mitsuhashi and M. Shimazu (1993) [Effect of temperature on mycelial growth of three muscardine fungi.] Trans. 44th Meet. Kanto Branch Jpn. For. Soc., 103-104 (in Japanese).

Shimazu, M. and H. Sato (1996) Media for selective isolation of an entomogenous fungus, Beauveria bassiana (Deuteromycotina: Hyphomycetes). Appl. Entomol. Zool. 31: 291-298. 